\title{
ANDROID BASED AUTONOMOUS COLOURED LINE FOLLOWER
}

\section{ROBOT}

\author{
Ramshetty K Sure ${ }^{1}$, Savita Patil $^{2}$ \\ ${ }^{1} P G$ Student, ECE Dept, AMC Engineering College Bangalore, Karnataka, India \\ ${ }^{2}$ Assoc. Prof, ECE Dept, AMC Engineering College Bangalore, Karnataka, India
}

\begin{abstract}
This paper introduces a Robot having the ability to choose a desired line among multiple coloured lines. Every line has different colors as their identities. The robot can differentiate among various colours and choose a desired one to find its target. Unlike any other simple line follower robot, this robot can be considered as a true autonomous line follower robot having the ability to detect presence of obstacle on its path. From the android mobile, instructions are given to the robot that senses a line and endeavours itself accordingly towards the desired target by correcting the wrong moves using a simple feedback mechanism but yet very effective closed loop system. The robot is capable of following very congested curves as it receives the continuous data from the sensors. This robot avoids collision and moreover, it can detect collision with obstacle sensor.
\end{abstract}

Keywords: IR Sensor, Photo Transistor, Obstacle Detector, Comparator, DC Motor, Microcontroller, Motor Driver, RFID Reader and Buzzer.

\section{INTRODUCTION}

Line follower robots are monotype mobile robot having the ability to follow a line very accurately which has an on board hardwired control circuit [1] and [2]. Usage of line follower robot is also increasing day by day. Mostly, in all industrial areas, where the automation is very much desired. A new kind of line follower robot can accelerate automatic transportation. Though, many researchers are studying regarding line follower robot but most of the concepts are concentrated on theoretical design. As a consequence a line follower robot is designed using artificial intelligence for autonomous driving and to stay on the line [3]. This robot team avoids collision among each other. Moreover, it is uncertain whether it can detect collision with obstacle or not. This design procedure is capable of tracking destination and avoids collision through sensors. A mobile robot controlling algorithm is developed having the ability of avoiding barriers [4]. Another algorithm for multiple controlling mobile robots is where prescribed in [5] where performance is shown also through simulation results. Most researchers preferred to show their designed robot performance through simulation. To meet the requirement of autonomous line based transportation, a new kind of line follower robot has to be designed. This paper provides an answer to that kind of requirements where there will be multiple destinations and the robot should have the ability to choose any of the desired destinations autonomously [6] and [7]. Moreover, the robot should have another ability of avoiding collision which can be achieved either by ultrasound or by infrared radiation. Without the application of complex sensor like camera and processors, this requirement is very difficult to fulfill. Neural Network can be used to track the target efficiently in a very short time [8] and [9] though it is not contributed in this paper. This robot is designed for practical applications which may include guidance system for industrial robots moving on shop floors, household applications or even at offices for transporting files from one room to any other room etc. [10].

In this paper section II describes the motivation of the system, where section III gives related work about this paper. Section IV describes the system overview, Section V deals with the motor interface and control hardware used in the robot, Section VI describes the robot control by android. Section VII gives the experimental results and finally section VIII concluded with electronics of the robot.

\section{MOTIVATION}

In order to reduce the human work, it is necessary of automation of color line following robot, this robot can be used in library to carry books from one place to another place, and it can be used for home automation, in restaurant it is used as robotic waiter. A restaurant in downtown Harbin, China, employs 20 robots instead of humans that cook, serve and entertain its guests. The restaurant was opened in June 2012, in this restaurant, line following robot is been used as a robot waiter running along tracks on the floor which carries the dishes to the desired table and it also serves dinner to the family. They are well trained, efficient and fast and they won't expect a tip for their efforts. This is 21 st century customer service; they can work continuously for five hours after a two- 
hour charge. But the worker will not work for free or a cheap labour - each robot costs between 200,000 to 300,000 hinese Yuan (US\$31,500 - US\$47,000) with an additional 5 million Yuan (US\$790,000) invested into the restaurant itself. China is expected to be the world's largest market for robots by 2014 [Source: arkazlive via YouTube].As the technology improves and the cost to build and run the robot drops. So large country like India, it is necessary to use of line following robot in restaurant, libraries, etc., but it is necessary to equip restaurant, library with colored line tracks.

\section{RELATED WORK}

In robotics there are many system invented which has different applications in different fields. Robotics is very popular field for research and manufacturing. Pakdaman M. et.al has design a small line following robot which used IR sensors to detect the line drawn on floor [1]. Priyank Patil has developed an AVR line following robot which can detect the line drawn on the floor with the help of sensor array. When its sensor is passing through the line drawn on the way then it reads 0 and vice versa [2]. That system has designed for the robot competition. Colak I. et.al has design a line following robot to use in the shopping malls for entertainment. That system used $4.8 \mathrm{~cm}$ wide black line to carry maximum load of $400 \mathrm{~kg}$. Two wheels balancing robot has developed by Nor Maniha Abdul Ghani et.al, which has the line following capability and for balancing it, they used infrared distance sensor to solve the problem in inclination [11], They also used a manual control with the help of remote controller [12]. A physical robot with 50 individual controls is generated by Gomi T. et.al from which the ability and gait to lift the body can be improved. That robot can move its legs in forward motion and tested in different conditions [13]. Roman Osorio C. et.al designed an intelligent line following robot, which can modify the performance of the movement with the help of different type of magnetic sensors. That robot was based on the V2X sensor which is a type of digital compass [14]. This system is used an array of 8 IR sensors and several LEDs. M. Zafri Baharuddin et.al designed a mobile robot which can be used as the navigation purpose [15]. An intelligent robot system is designed by Bajestani S. E. M. which can give corrective feedback in different colors of light [16]. They used a comparator circuit to improve the sensitivity of the system. That comparator compares the voltage with the predetermined amounts from which a robot can move in accurate real time.Kazi Mahmud Hasan et.al designed sensor based autonomous color line following robot with obstacle avoidance, This robot can follow not only black and white colors but also some other different colors. This robot includes electronic logic gates as brain instead of microcontroller [17].

\section{SYSTEM OVERVIEW}

The proposed system consists of a PIC controller interfaced with three IR sensors for detecting the direction of movement for the robot. These sensors will be detecting the colour difference between the black and white for the smooth following of line. Further, an obstacle detector prevents the robot from hitting any obstacles by stopping the robot once any object is detected. The Controller takes action on the feedback from the sensors and accordingly the motors are controller for forward, reverse or turning movements. The destinations in the track for robot are detected by using coloredIR sensor, this sensor detecting green, blue, red and yellow. The Android mobile sends commands to the PIC Microcontroller using Bluetooth and UART communication. The controller then takes action depending on the command and directs the robot into the required Path. The LED and phototransistor based sensors are used here to sense the line. Four LEDs (TX) and phototransistor sensors (Rx) facing the ground has been used in this setup.

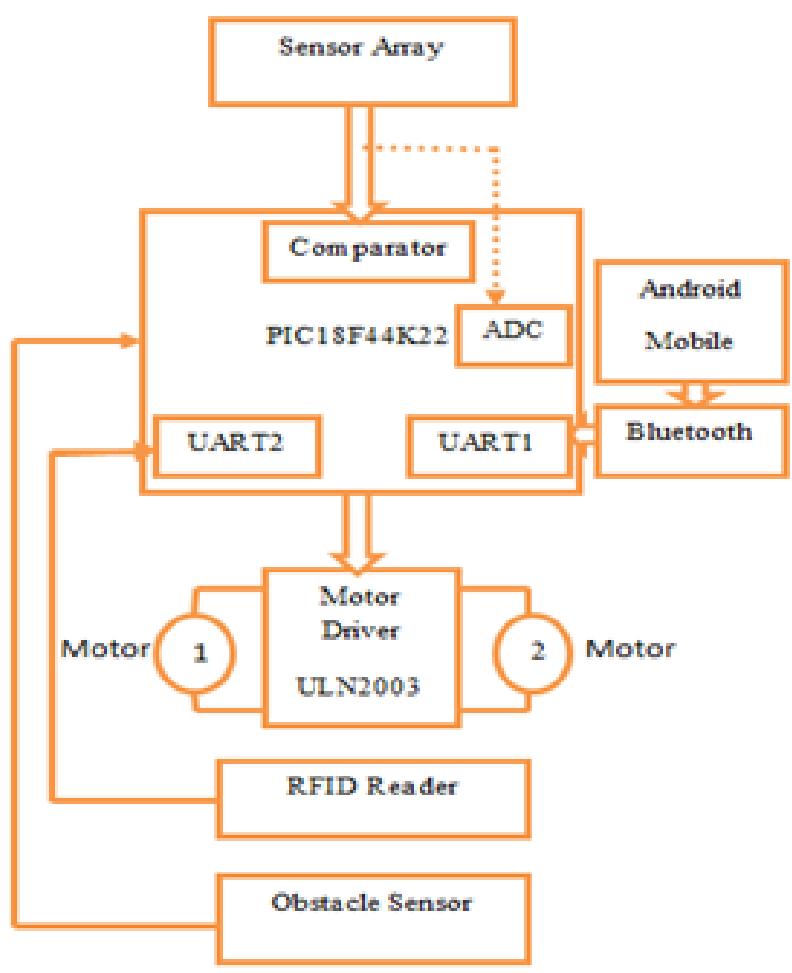

Fig-1: Block diagram of colour line follower robot.

Two of them are used as line detectors and rest of them is used for detecting destination. The output of the sensors is analog in nature which depends on the amount of light reflected back. This analog signal is then processed to produce digital data containing information about the line and color. An IR obstacle sensor is used to gather information about the nearby obstacle present, which may block the line. Processing all those data from the installed sensors, the robot's brain generates control signals automatically to perform the desired movements. Suppose ambient light interfacing with sensor, it may disturb the transition of data at that time. Sensors are not going to identify the color lines. In these conditions RFID 
reader detect the desired target by detecting RFID tag placed at the destination. RFID reader reads the desired tag only when the instructions are given to the robot through android mobile.

\subsection{Sensors}

The robot uses two different types of sensors to gather all the necessary information. The sensors are line sensor and IR obstacle sensor.

\subsubsection{Line Sensor}

An infrared emitter is an LED made from gallium arsenide, which emits near infrared energy at about $880 \mathrm{~nm}$. The infrared phototransistor acts as a transistor with the base voltage determined by the amount of light hitting the transistor. Hence, it acts as a variable current source. Greater amount of IR light cause greater currents to flow through the collectoremitter leads. The phototransistor is wired in a similar configuration to the voltage divider. The variable current traveling through the resistor causes a voltage drop in the pullup resistor. This voltage is measured as the output of the device

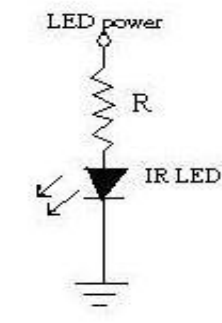

An IR emitter

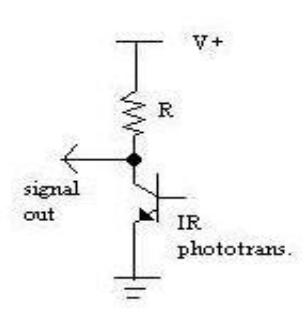

An IR phototransistor
Fig-2: circuit diagram of infrared sensor

IR reflectance sensors contain a matched infrared transmitter and infrared receiver pair. These devices work by measuring the amount of light that is reflected into the receiver. Because the receiver also responds to ambient light, the device works best when well shielded from ambient light and when the distance between the sensor and the reflective surface is small.IR reflectance sensors are often used to detect white and black surfaces. White surfaces generally reflect well, while black surfaces reflect poorly. All the other colors have voltage drops in between white and black color. A good sensor will be able to differentiate different colors even if the separation between two colors is very small. A powerful logical equation is developed to utilize this property to extract color information and build this line follower robot. To reduce the ambient light interference, the line sensors are placed in a bounded region where no ambient light can interfere in getting data.

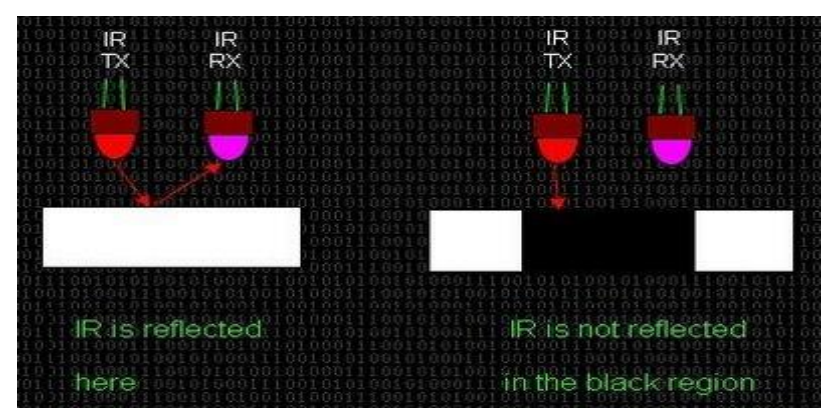

Fig-3 Line sensor arrangement and working

\subsubsection{Obstacle Sensor}

An IR sensor is used for detecting presence of an obstacle. The sensor consists of an IR transmitter and receiver. There is an IR LED

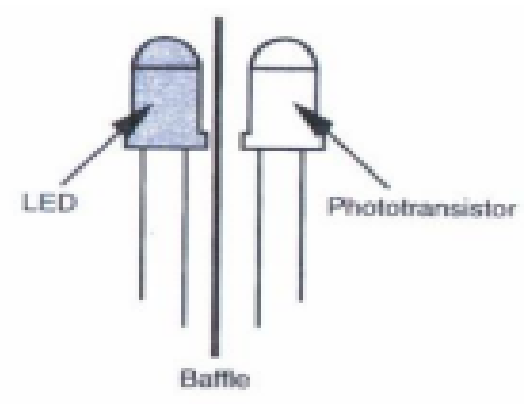

Fig-3: Obstacle Sensor

which emits IR light in front of the robot and if there is any obstacle nearby which blocks the line, the receiver of the IR sensor picks up the reflected IR light and produces a logic ' 1 ' which then forces the robot to stop. Otherwise the output of the IR sensor is logic ' 0 '. The IR transmitter modulates the IR light to produce IR pulses at $38 \mathrm{KHz}$ so that ambient light cannot create any interference in detecting presence of obstacle.

\subsection{Comparator}

Comparator is use to make the system sensitive as per the requirement. It usually compares the voltages between the inverting and non-inverting terminals. A threshold voltage is set on the reference voltage in the operational amplifier in inverting or non-inverting terminal. If another terminal voltage that is input voltage is greater than this threshold voltage then it gives the output. And if the input voltage is less then threshold voltage then it cannot gives any output. The sensitivity of the sensor can be varying by set a threshold voltage in the comparator circuit. By using this circuit a Phototransistor can be used for low beam of light and an IR sensor for detect the obstacle from a large distance. 


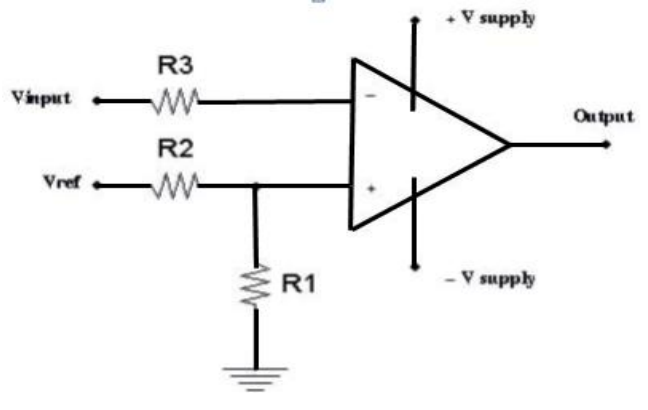

Fig-4: Comparator circuit.

\subsection{RFID Reader}

RFID is a method of identifying unique items using radio waves. It includes readers, antenna, mixed signal IC, RF receiver and RF transmitter, Software based communication protocols and networking. A non-contact system that can monitor and track items or individuals. Provide unique identification that allows for a wide range of applications Perform the operation using unobtrusive, "low cost" components. Use Wireless Communications techniques to facilitate the system design. Main perpuos of radio frequency identifier in this paper, suppose ambient light interfacing with sensor, it disturb the transitions of data at that time sensors are not going to identify the color lines. In these conditions RFID reader detect the desired target by detecting RFID tag placed at the destination. RFID reader reads the desired tag only when instructions given to the robot through android mobile.

\subsection{Algorithm}

In start microcontroller check the sensor unit or receiver unit. Then verifies this address if it is correct then it continue process else it waits for appropriate address. If address is correct then it moves towards sensor array. If center sensor activate then all motors rotates and line follower move forward. Else if right sensor activates then motors rotates in manner that line follower move toward right. Else if left sensor activate then line follower move towards left. Else if left, right and center sensor activate at same time then all motors stop and transmit signal. It stops till not received appropriate address signal. For destination RFID reader or color sensor address matches then robot stop for that position else moves forward to other position

\section{MOTOR INTERFACE AND CONTROL}

\section{HARDWARE}

\subsection{Motor Driver}

Motor driver act like the current amplifier. It is use for controlling the current in the motor. The motor drive provides high current as the dc motor need when it receives low current in the circuit. For drive the motors a high value of the current is needed. ULN2003 IC can control the two dc motor simultaneously. It can rotate the motor in the forward and reverse direction. By using the motor driver a line following robot can be move in clockwise and in anticlockwise directions. It completely controls the movement of the $\mathrm{dc}$ motor that's why it has been called as motor driver.

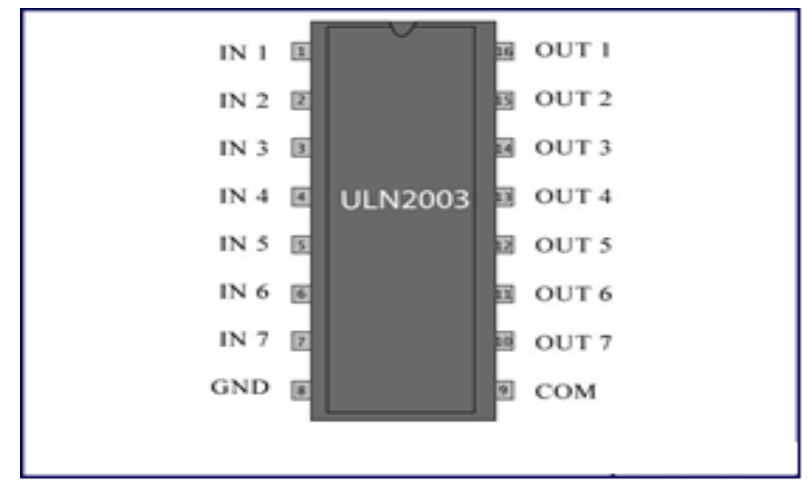

Fig-5: Motor driver IC.

\subsection{Motors and Wheels}

For the proper movement of the system two dc motors has been used in the circuit and a castor wheel is attached in the front side of that Line Following Robot. Caster wheel enable the movement of the robot is easy in every direction. Two dc motors at the end side of the robot is controlled by the motor driver. For controlling the complete system a microcontroller is used, which set its flag bit as per the different situations. And this complete system need a small power supply of 6$12 \mathrm{~V}$, which can be provided form a battery. That is why this system is cost effective and operates in very low power supply.

\subsection{Voltage Regulator}

This LM7805 series of fixed-voltage integrated-circuit voltage regulators is designed for a wide range of applications. These applications include on-card regulation for elimination of noise and distribution problems associated with single-point regulation. Each of these regulators can deliver up to $1.5 \mathrm{~A}$ of output current. The internal current-limiting and thermalshutdown features of these regulators essentially make them immune to overload. In addition to use as fixed-voltage regulators, these devices can be used with external components to obtain Adjustable output voltages and currents, and also can be used as the power-pass element in precision regulators.

\subsection{Comparators, Voltage Divider and Controller}

Color separation requires comparator and controller for their implementation.Voltage divider networks provide necessary reference voltages. Controller act as brain of the robot which makes it extremely cost effective and reduces complexity. 


\section{ANDROID CONTROL}

Android is a mobile operating system that is based on modified version of Linux. Android applications developed using tools such as JDK, Android SDK, eclipse and Android development tool. Architecture of Android consists of four layers such as Linux kernel, Libraries, Application framework, Applications. Kernel layer contains all the low level device drivers for the various hardware components of an Android device, Libraries contain all the code that provides the main features of an Android OS. For example, the SQLite library provides database support so that an application can use it for data storage. The Web Kit library provides functionalities for web browsing. Applications layer is top layer, here we will find applications that ship with the Android device (such as Phone, Contacts, Browser, etc.), as well as applications that we download and install from the Android Market.

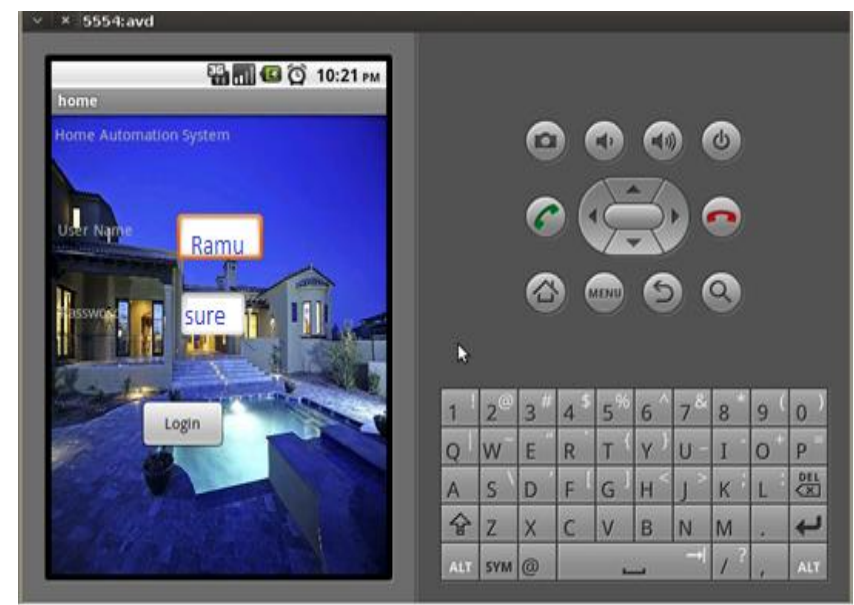

Fig-6: Main Menu of Robot control System

From the above control system, instructions are given to the robot to reach its desired destination.

\section{EXPERIMENT RESULTS}

Phototransistor is basically acting as a variable current source which changes with different intensity of light. With the help of comparator circuit it can give the output when it comes under the contact of that line drawn. By using motor driver it follows the line drawn on the floor. At the turn of the line drawn, phototransistor doesn't get any output. DC motor must be controlled by the motor driver for movement of the robot. For left movement the left side DC motor should be stopped and the right side DC motor should be run in forward direction. Table I shows the different necessary conditions for the movements of the motor. When the system detects any obstacle in its path then the DC motor stops its rotation and a buzzer is activated with the help of microcontroller unit. Microcontroller can be used for controlling the Robot system in every possible case.
Table-1: Direction movement of robot

\begin{tabular}{|l|l|l|}
\hline $\begin{array}{l}\text { ROBOT } \\
\text { MOVEMENT }\end{array}$ & LEFT MOTOR & RIGHT MOTOR \\
\hline Straight & Straight & Straight \\
\hline Left & Stop & Straight \\
\hline Sharpe Left & Reverse & Straight \\
\hline Right & Straight & Stop \\
\hline Sharpe Right & Straight & Reverse \\
\hline Reverse & Reverse & Reverse \\
\hline
\end{tabular}

Different paths for robot movement as showing in figure 7 interfacing of the microcontroller with the sensors and RFID reader is shown in the figure 8 and also a snapshot of the color line following robot with the line drawn on the floor as shown in the figure 9 .

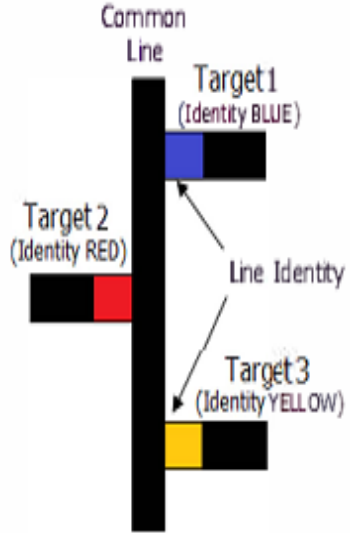

(a)

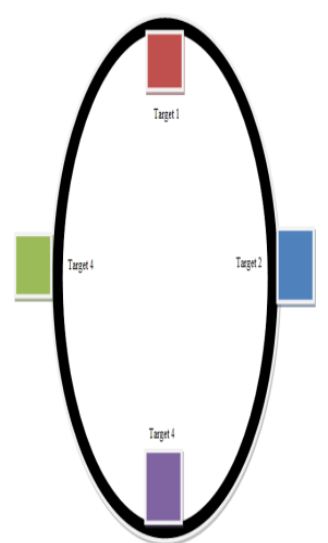

(b)
Fig-7: Different paths for robot movement

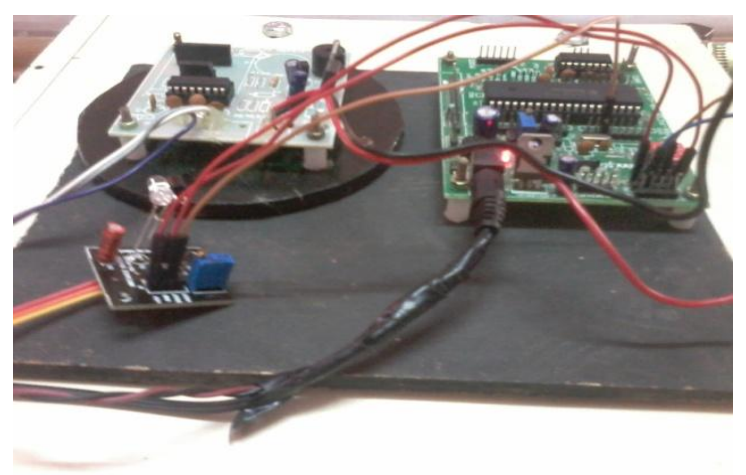

Fig-8: Microcontroller with RFID Reader and sensor interface 


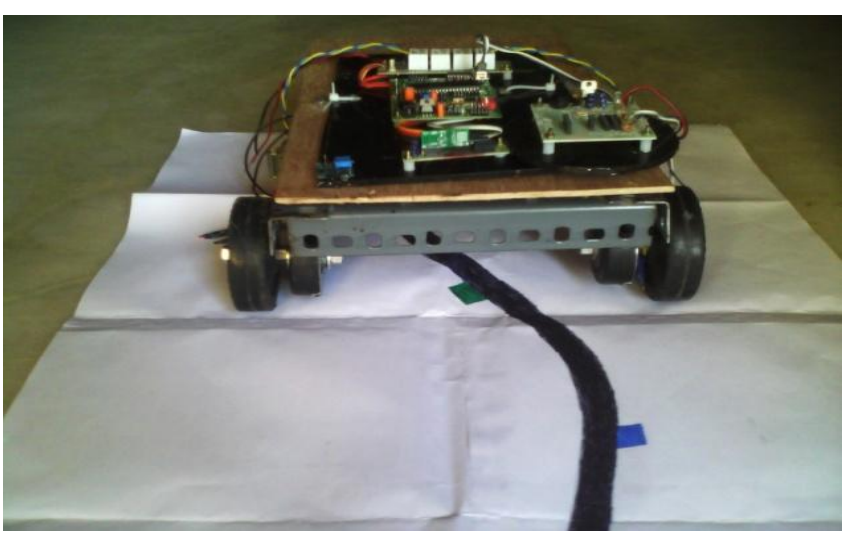

Fig-9: Snapshot of color line following robot

\section{CONCLUSIONS}

The concept of the android based colour line follower robot is practically implemented in this paper based on PIC Microcontroller, RFID reader and sensors. Simultaneously it makes the use of instructions from sensors and on board PIC Microcontroller which performs the physical movements. The robot is succeeded to locate and follow the target by giving instruction through android mobile. This robot can follow not only black and white colors but also some other different colors. If the conditions are properly set and calculations are accurately done, then performance rate and accuracy will be more than ever. Further, modification of this robot includes application of shortest path algorithm and neural network so that it can find its target more efficiently in shortest amount of time.

\section{REFERENCES}

[1]. Pakdaman,M.Sanaatiyan,M.M.,"Design and Implemen tation of Line Follower Robot,", Second International Conference on Computer and Electrical Engineering TCCEE '09, vol.2, pp.585-590,Dec.2009.

[2]. PriyankPatil,"AVR Line Following Robot," Department of Information Technology K. 1. Somaiya College of Engineering Mumbai, India. Mar 5, 2010.

[3]. Dean A. Pomerleau; Jay Gowdy; Charles E. Thorpe, "Combining Artificial Neural Networks and Symbolic Processing for Autonomous Robot Guidance," Engng Applic. Artif. Intell. Vol. 4, No. 4, pp. 279-285, 1991.

[4]. L1U Shi-Cai,L1U Guang-Jun, "Formation Control of Mobile Robots with Active Obstacle Avoidance," Acta Automatica Sinica, Vol. 33, No. 5, 2007.

[5]. YANG Tian-Tian; L1U Zhi-Yuan; CHEN Hong; PEl Run, "Formation Control and Obstacle Avoidance for Multiple Mobile Robots," Acta Automatica Sinica, Vol. 34, No. 5, 2008.

[6]. Sandhana, Lakshmi (2002-09-05), “A Theory of Evolution for Robots, "Wired Magazine ... Retrieved 2007$10-28$.
[7]. J.Kramer and M. Scheutz, "Development environments for autonomous mobile robots: A survey, "Autonomous Robots, vol. 22.

[8]. Floreano, D.,Mondada F.,"Evolutionary neurocontrollers for autonomous mobile robots," Neural Networks 11, pp. 1461-1478, 1998.

[9]. Hagras,H., Pounds-cornish, A., Colley, M., Callaghan, V.,Clarke,G.: Evolving Spiking Neural Network Controllers for Autonomous Robots. Proceedings of the 2004 IEEE International Conference on Robotics and Automation, pp. 4620-4626., 2004

[10]. Charles A. Schuler, Willam L. McNamee, "Industrial Electronics and Robotics," McGraw-Hill International Edition, Industrial Electronics Series 2003.

[11]. Nor Maniha Abdul Ghani, FaradilaNaim, Tan Pi own yon, "Two Wheels Balancing Robot with Line Following Capability ".

[12]. Colak, I., Yildirim, D.,"Evolving a Line Following Robot to use in shopping centers for entertainment", Industrial Electronics, 2009. IECON '09. 35th Annual Conference of IEEE, pp.3803 - 3807, 3-5 Nov. 2009.

[13]. T. Gomi, K. Ide, "Evolution of gaits of a legged robot", IEEE International Conference on Fuzzy Systems 06/1998; DOI:10.1109/FUZZY.1998.687476 ISBN: 0-7803-4863-X In proceeding of: Fuzzy Systems Proceedings, 1998.

[14]. Román Osorio," Intelligent Line Follower Mini-Robot System", International Journal of Computers, Communications \& Control Vol. I, No.2, pp.73-83, 2006.

[15]. M. Zafri Baharuddin, Izham Z. Abidin, S. Suleiman Kaja Mohideen, Yap Keem Siah, Jeffrey Tan Too Chuan,"Analysis of Line Sensor Configuration for the Advanced Line Follower Robot", University Tenaga Nasional.

[16]. Bajestani, S.E.M., Vosoughinia, A., "Technical Report of Building a Line Follower Robot" International Conference on Electronics and Information Engineering (ICEIE 2010), vol 1, pp. v1-1 v1-5, 2010.

[17]. Kazi Mahmud hasan, Abdullah -nahid,k. Reza,s.khatun and m. R.basar"Sensor Based Autonomous Color Line Follower Robot with Obstacle Avoidance"2013 IEEE Business Engineering and Industrial Application Colloquium (BEIAC). 